NBER WORKING PAPER SERIES

\title{
QUANTIFIER ELIMINATION FOR DEDUCTION IN ECONOMETRICS
}

\author{
Casey B. Mulligan \\ Working Paper 24601 \\ http://www.nber.org/papers/w24601
NATIONAL BUREAU OF ECONOMIC RESEARCH
1050 Massachusetts Avenue
Cambridge, MA 02138
May 2018

This work has benefitted from discussions with Clark Barrett, Nikolaj Bjorner, Russell Bradford, James Davenport, Matthew England, Lars Hansen, Leo de Moura, Zak Tonks, Alex Torgovitsky, comments from minicourse participants at Chicago, LSE, Tel-Aviv University, and Stanford, and the research assistance of Chanwool Kim. The views expressed herein are those of the author and do not necessarily reflect the views of the National Bureau of Economic Research.

NBER working papers are circulated for discussion and comment purposes. They have not been peer-reviewed or been subject to the review by the NBER Board of Directors that accompanies official NBER publications.

(C) 2018 by Casey B. Mulligan. All rights reserved. Short sections of text, not to exceed two paragraphs, may be quoted without explicit permission provided that full credit, including (C) notice, is given to the source. 
Quantifier Elimination for Deduction in Econometrics

Casey B. Mulligan

NBER Working Paper No. 24601

May 2018

JEL No. C01,C63,C65

\begin{abstract}
When combined with the logical notion of partially interpreted functions, many nonparametric results in econometrics and statistics can be understood as statements about semi-algebraic sets. Tarski's quantifier elimination (QE) theorem therefore guarantees that a universal algorithm exists for deducing such results from their assumptions. This paper presents the general framework and then applies QE algorithms to Jensen's inequality, omitted variable bias, partial identification of the classical measurement error model, point identification in discrete choice models, and comparative statics in the nonparametric Roy model. This paper also discusses the computational complexity of real $\mathrm{QE}$ and its implementation in software used for program verification, logic, and computer algebra. I expect that automation will become as routine for abstract econometric reasoning as it already is for numerical matrix inversion.
\end{abstract}

Casey B. Mulligan

University of Chicago

Department of Economics

1126 East 59th Street

Chicago, IL 60637

and NBER

c-mulligan@uchicago.edu 
Econometrics has been profoundly affected by progress in information technology that has facilitated the collection and processing of vast amounts of data related to economic activity. Deducing theoretical conclusions remains critical in almost every field of the profession, but so far has received less assistance from technology. There are automatic algebraic simplifiers, but simplicity is often in the eye of the beholder. Computers have already been used for generating numerical examples and Monte Carlo simulations, but approximation quality is a concern, and more thinking is always needed to appreciate the generality of the results from examples. The purpose of this paper is to show how approximation-free econometric reasoning is beginning to be automated, present the mathematical foundations of those procedures, and allow readers of this paper to access a user-friendly tool for automated reasoning.

Deductive reasoning can be described as a process of quantifier elimination (QE), and has been described that way by mathematicians and logicians since the nineteenth century. ${ }^{1}$ Merely as a way of describing reasoning rather than an alternative engine for doing it, QE has historically been of little interest in economics, statistics, and related fields. However, mathematics and computer science have more recently invented, improved and implemented algorithms for quantifier elimination and thereby methods for automated reasoning. Section I therefore introduces, to an econometrics audience, quantified systems of polynomial equalities and inequalities, and their quantifier-free equivalents, as defined in real algebraic geometry. Section II notes the parallels between $\mathrm{QE}$, projection, and the satisfiability problem in computer science. These parallels are helpful for discovering ways that $\mathrm{QE}$ can be used in econometric analysis.

At first glance, automated QE methods appear too specialized for many econometrics problems, especially the nonparametric ones, because their polynomial structure can be subtle. Here the notion of partially interpreted functions is especially helpful for discovering that structure and therefore making use of automated QE. Section III introduces partially interpreted functions and uses them to apply QE to omitted variable bias, partial identification of the classical measurement error model, comparative statics in the nonparametric Roy model, and point identification in discrete choice models. In other words, the polynomial framework is not nearly as restrictive as it first

\footnotetext{
${ }^{1}$ DeMorgan (1862) is an early reference.
} 
appears. Results from mathematicians Tarski, Collins, and followers - shown in Section IV - speak to the feasibility of, and algorithms for, eliminating quantifiers from systems of polynomial equality, inequality, and not-equal relations (hereafter "polynomial inequalities") and thereby for confirming, refuting, and developing many hypotheses in econometrics. Section V points readers to existing software implementations of QE methods.

This is the first paper to use automated QE or nonlinear software-verification methods to deduce conclusions for econometric models. ${ }^{2}$ To my knowledge, even the mathematics and computer science literatures have yet to treat integrals or vector dot products as partially interpreted functions for the purpose of applying QE or softwareverification methods; this treatment dramatically broadens the scope of applicable econometric models. Mulligan (2016) treats utility and production functions as partially interpreted functions and uses QE algorithms to reach conclusions in economic theory. While noting that it can be useful to "decide whether or not a given semialgebraic set is contained in another one" (Kauers 2011, p. 2), those literatures give little specific attention to the assumption-hypothesis framework emphasized here. That framework reveals how QE is a tool for, among other things, recovering missing assumptions or discovering True hypotheses. Before Mulligan (2016), QE or related methods had been discussed, and once implemented computationally (Li and Wang 2014), in the economic theory literature discussed below, especially as relates to economies with utility functions that are polynomial in the commodities.

\footnotetext{
${ }^{2}$ Software-verification tools have been used to check the software running auctions (Dennis, et al. 2012) and financial algorithms (Passmore and Ignatovich 2017) and to confirm that auctions are strategy proof (Tadjouddine, Guerin and Vasconcelos 2009). Auctions and social choice problems have also been studied with higher-order logic proof assistant software (Kerber, Lange and Rowat 2016), which falls outside the Tarski framework used in this paper and requires users to manually guide the proof environment and strategy.
} 


\section{Sets and hypotheses represented with and without quantifiers}

\section{I.A. Semi-algebraic sets defined with quantifiers}

The general framework has $N<\infty$ real scalar variables $x_{1}, \ldots, x_{N}$. A quantified representation of a set, $Q R$, is a "Tarski formula" in the $N$ variables with $N-F$ of them quantified and the remaining $0 \leq F<N$ free (unquantified):

$$
\begin{gathered}
Q R=\left(Q_{1} x_{1}\right)\left(Q_{2} x_{2}\right) \ldots\left(Q_{N-F} x_{N-F}\right) T\left(x_{1}, x_{2}, \ldots, x_{N}\right) \\
Q_{i} \in\{\forall, \exists\} i=1, \ldots,(N-F)
\end{gathered}
$$

where any "Tarski formula" $T$ by itself is a quantifier-free Boolean combination, with the logical And $(\wedge)$ and Or $(\vee)$ operators, of a finite number of polynomial (in $x_{1}, \ldots, x_{N}$ ) inequalities. ${ }^{3}$ For brevity I also use the Negation $(\neg)$ operator, which merely refers to reversing an inequality (or changing $=$ to $\neq$ ), and the Implies $(\Rightarrow)$ operator, which is a shorthand for a Boolean combination of Or and Not. ${ }^{4}$ There are two possible quantifiers: existential "Exists" $(\exists)$ and universal "ForAll" $(\forall)$.

Of particular interest are universal and existential formulations that have the same quantifier on each of the $N-F$ variables. In these cases, I show the quantifier only once and list the quantified variables in braces:

$$
\left(Q x_{1}\right)\left(Q x_{2}\right) \ldots\left(Q x_{N-F}\right) T\left(x_{1}, x_{2}, \ldots, x_{N}\right) \equiv Q\left\{x_{1}, x_{2}, \ldots, x_{N-F}\right\} T\left(x_{1}, x_{2}, \ldots, x_{N}\right)
$$

Hypotheses formulated with only one kind of quantifier, e.g., (2), have the same meaning regardless of the order of the quantifiers. Moreover, every universal formulation can be expressed as an existential formulation, and vice versa: ${ }^{5}$

$$
\neg \forall\left\{x_{1}, x_{2}, \ldots, x_{N-F}\right\} T\left(x_{1}, x_{2}, \ldots, x_{N}\right)=\exists\left\{x_{1}, x_{2}, \ldots, x_{N-F}\right\} \neg T\left(x_{1}, x_{2}, \ldots, x_{N}\right)
$$

\footnotetext{
${ }^{3}$ C.W. Brown (2004, 2). The set represented by a Tarski formula is known as semi-algebraic.

${ }^{4} A \Rightarrow H$ is equivalent to $\neg A \vee H$.

5 (3) is known as "De Morgan's law for quantifiers."
} 
If, for given values of the free variables, the Tarski formula is not True on all of $\mathbb{R}^{N-F}$, then there exists at least one point in $\mathbb{R}^{N-F}$ where the Tarski formula is False, and vice versa. The order-invariance and quantifier-interchangeability properties of universal and existential formulations offer many opportunities for facilitating and verifying computation.

\section{I.B. Quantifier elimination and deductive reasoning}

We are interested in a quantifier-free representation of the same set $Q R$. Formally,

$$
Q R=P\left(x_{N-F+1}, \ldots, x_{N}\right)
$$

where $P$ is another Tarski formula (distinct from the $T$ appearing in $Q R$ ), and therefore a quantifier-free Boolean combination of a finite number of polynomial inequalities. Real quantifier elimination $(\mathrm{QE})$ refers to an algorithmic method that derives $P$ from $Q R$. The existence of such a $P$, and the existence of a single quantifier-elimination algorithm applicable to all quantified formulae, is guaranteed as a special case of Tarski's famous proof. $Q R$ is therefore a semi-algebraic set, too. ${ }^{6}$

Because the quantified variables are absent from the result of $Q E, Q E$ is sometimes called "eliminating a variable" (from a system of polynomial inequalities), especially when the quantifiers are existential. ${ }^{7}$ If there are no free variables $(F=0), Q R$ is known as a "sentence" and $P$ must be either $1=1$ (True) or $1=0$ (False). A decision method is a quantifier-elimination method for sentences: it decides whether a sentence is True or False.

Deductive reasoning in econometrics and other fields involves deciding whether a hypothesis $H$ is implied by a set of assumptions $A$. If $H$ and $A$ were each semi-algebraic sets, then we could decide two existential sentences:

\footnotetext{
${ }^{6}$ Tarksi made the proof in 1930 (Caviness and Johnson 1998, p. 1), but the result was not published until Tarski (1951)

${ }^{7}$ Another branch of elimination theory deals with equations only, where Grobner-basis methods are frequently used (Cox, Little and O'Shea 2007).
} 
(i) Does there exist an example: a point $v \in \mathbb{R}^{N}$ that is in both $A$ and $H$ ?

(ii) Does there exist a counterexample: a point $v \in \mathbb{R}^{N}$ that is in both $A$ and $\neg H$ ?

where I use $v$ here and throughout the paper to denote a vector, as distinct from scalars that are denoted $x_{1}, x_{2}$, etc. Four results are possible, as summarized in Table 1. Should technology provide these two decisions automatically then the econometrician gains important information: either a confirmation or refutation of his theory, or the knowledge that his assumptions contradict. ${ }^{8}$

QE can do even more. It can generate True hypotheses when the econometrician has not devised one on his own, by partitioning $v$ into free and bound variables, $f$ and $b$, respectively, and eliminating quantifiers from $\exists b[A(f, b)]$. The result is a formula $P(f)$ in the free variables that is a hypothesis deduced from $A .{ }^{9}$

QE is especially useful in the mixed case, where by definition the assumptions $A$ are insufficient. Here we can eliminate quantifiers from the set of counterexamples $\exists b[A(f, b) \wedge \neg H(f, b)]$, which gives us a formula $P(f)$ in the free variables. Because $P(f)$ must be True for all counterexamples, $\neg P(f)$ must rule out all counterexamples. In other words, conditional on the assumptions $A, \neg P(f)$ is sufficient to deduce the hypothesis: $\forall\{b, f\}[A(f, b) \wedge \neg P(f) \Rightarrow H(f, b)]=$ True.

Specific QE algorithms, discussed further below, have byproducts that further assist deductive reasoning. As noted above, $\neg \exists b[A(f, b) \wedge \neg H(f, b)]$ is conditionally sufficient for $H$, but not necessary. A necessary and sufficient condition is $A(f, b) \wedge$ $H(f, b)$, which can be informative if represented by a recursive, quantifier-free formula, which is exactly what is constructed by the Cylindrical Algebraic Decomposition (CAD)

\footnotetext{
${ }^{8}$ Because of their relationship with proofs, deciding sentences $(F=0)$ is especially useful for automating reasoning. This contrasts with previous discussions of quantifier elimination in economic theory, such as Brown and Matzkin (1996), Snyder (2000), Brown and Kubler (2008), Carvajal et al. (2014), and Chambers and Echenique (2016), whose purposes are to derive restrictions on free variables that they associate with "observables." Moreover, with an exception appearing in the appendix of Brown and Matzkin (1996), they do not intend to "carry out" the quantifier elimination but rather be assured that the result of doing so would be a non-empty semi-algebraic set in $\mathbb{R}^{F}$.

${ }^{9}$ By construction, there can be no counterexample. To see this, suppose otherwise: $\exists\{f, b\}[A(f, b) \wedge \neg P(f)]=\exists f\{\exists b[A(f, b) \wedge \neg P(f)]\}$. But if the intersection of those two sets is not empty, then neither set can be empty by itself: $\exists f\{\exists b[A(f, b)] \wedge \exists b[\neg P(f)]\}=$ $\exists f\{[P(f)] \wedge[\neg P(f)]\}$, which is impossible because $P$ and $\neg P$ cannot be True at the same time.
} 
algorithm for QE. Many algorithms for deciding existential sentences automatically provide examples for True sentences, which means that in Table 1's mixed case we would have an "example" point $v \in \mathbb{R}^{N}$ that is in $A \wedge H$ and another "counterexample" point that is in $A \wedge \neg H .^{10}$ All of these uses of QE are illustrated below with specific examples from econometrics.

These are some of the reasons why it can be of "enormous" practical value to "eliminate quantifiers" from a set's definition: that is, to take a quantified definition of the form (1) and transform it into a quantifier-free one such as the Tarski formula $P$ on the RHS of (4). ${ }^{11}$ Indeed, some artificial intelligence research equates quantifier elimination with the vernacular concept of "solving" a mathematics problem (Arai, et al. 2014, p. 2).

\section{QE, Projection, and Satisfiability: Illustrated with Jensen's Inequality}

\section{II.A. Set Projection as QE}

Removing existential quantifiers from the formula defining a set in $\mathbb{R}^{N}$ is the algebraic equivalent of projecting that set into the space of free variables. If there are no free variables, then the decision or quantifier elimination is the algebraic equivalent of projecting the set onto the origin. Specifically, an empty set has no projection on the origin (False) and a nonempty set has a projection on the origin (True).

Consider the well-known result that the expectation of the square of a random variable with positive variance exceeds the squared expectation of that variable. This toy example is unusual in exhibiting an obvious polynomial structure, but that serves the

\footnotetext{
${ }^{10}$ Any QE algorithm is a useful tool for generating an example point from a semi-algebraic set. Existentially quantify $N-1$ of the variables in the Tarski formula leaving free, say, $x_{1}$, and then eliminate quantifiers. The result is a formula in $x_{1}$ alone. Choose a real number for $x_{1}$ that satisfies the formula and substitute that value into the original $N$-variable Tarski formula, making it an $(N-1)$-variable Tarski formula. Repeat the process for $x_{2}$, etc., until real numbers are assigned to all $N$ variables.

${ }^{11}$ Caviness and Johnson (1998, p. 2).
} 
purpose at the moment to further illustrate QE with free variables and relate it with projection. In the two-state case, the result says:

$$
\begin{gathered}
\neg \exists\left\{p, x_{1}, x_{2}\right\} \\
{\left[0<p<1 \wedge x_{1} \neq x_{2}\right] \wedge\left[\left(p x_{1}^{2}+(1-p) x_{2}^{2}\right) \leq\left(p x_{1}+(1-p) x_{2}\right)^{2}\right]} \\
=\text { True }
\end{gathered}
$$

The assumption-hypothesis pair shown in (5) fits into the True entry of Table 1. In general, a hypothesis can be deduced from a set of assumptions if and only if the set of counterexamples is empty. That set is empty if and only if it has no projection on the origin.

In order to adapt this example to the mixed case in Table 1, let's drop the assumption $p<1$. Now the Tarski formula for a counterexample is:

$$
\left[0<p \wedge x_{1} \neq x_{2}\right] \wedge\left[\left(p x_{1}^{2}+(1-p) x_{2}^{2}\right) \leq\left(p x_{1}+(1-p) x_{2}\right)^{2}\right]
$$

which is True for some values of $\left\{p, x_{1}, x_{2}\right\}$ but False for others. The three-dimensional set of counterexamples described by (6) is shown in Figure 1. We can use projection/QE to discover the missing assumption by eliminating existential quantifiers from (6), leaving $p$ as a free variable:

$$
\begin{gathered}
\neg \exists\left\{x_{1}, x_{2}\right\}\left\{\left[0<p \wedge x_{1} \neq x_{2}\right] \wedge\left[\left(p x_{1}^{2}+(1-p) x_{2}^{2}\right) \leq\left(p x_{1}+(1-p) x_{2}\right)^{2}\right]\right\} \\
=p<1
\end{gathered}
$$

In geometric terms, the projection of the counterexamples set onto the $p$ axis is $p \geq 1$, which means that counterexamples are ruled out by $\neg(p \geq 1)=p<1$. In logical terms, the $\mathrm{QE}$ in (7) generates a new assumption $p<1$ that, in combination with the previous ones, rules out all counterexamples, regardless of the values of $x_{1}$ and $x_{2}$. 


\section{II.B. Satisfiability as QE}

Deciding existential and universal sentences is an important special case of QE, and is closely related to the Boolean satisfiability problem in computer science, which is whether there are any values of the Boolean variables in a formula that make the entire formula True. The inequality atoms of a Tarski formula are Boolean valued (an inequality is either satisfied or not), but with the added complexity that the Boolean values in different parts of the formula are related to the extent that the inequalities contain the same scalar variables. This and related extensions to the satisfiability problem recently have been developed in the field of satisfiability modulo theories (SMT). The specific theory of interest here is the theory of arithmetic (addition, subtraction, multiplication) on real numbers, known as "nonlinear real arithmetic" (NRA) in the SMT field (Jovanović and de Moura 2012).

Automated SMT solvers with NRA capabilities are actively being developed (see www.SMT-LIB.org, and section V below) in the public domain and by major software companies, especially for the purpose of verifying complicated hardware and software systems. System inputs from external sources are represented as scalar or Boolean variables and the Tarski formula describes a set of potential unintended results of ("bugs from") the computation on those inputs. The hardware or software developer uses the SMT solver to obtain a guarantee that those bugs cannot occur. Because they are just deciding existential sentences of the type described in subsection I.B, SMT-NRA solvers can serve as engines for automated econometric reasoning at least to the extent that no free variables are needed. In this way real algebraic geometry (projection) and computer science (SMT-NRA) are tackling essentially the same automated reasoning problem from different perspectives. 


\section{Using partially-interpreted functions to recognize instances of real quantifier elimination in econometrics}

We are also interested in the existence and properties of automated QE method(s), but first we consider some familiar hypotheses from econometrics. At first glance many econometrics examples do not appear to fit into the framework (1), (4) because their polynomial structure is not obvious. But the theoretical computer science notion of a partially interpreted function helps reveal that structure. The integration operator (on integrable functions) is an important example for econometric analysis. Another example is the vector dot product, which becomes a partially interpreted function when we add Gramian matrix restrictions to the assumption set. See also Mulligan (2016), which shows how the utility and production functions used in economic theory are also usefully understood as partially interpreted functions.

\section{III.A. An introduction to uninterpreted functions}

So far I have used integer indices to distinguish one scalar, say $x_{1}$, from another such as $x_{2}$. Although the variables in a Tarski formula must be scalars, nothing requires that the indices be scalars. The indices could be, say, names, or natural language words, or images. Or the indices could be integrable functions, as in the Roy model below, or arbitrary-length vectors as in the sections that follow. In other words, the variables in a Tarski formula can be points on any abstract mapping from objects (vectors, integrable functions, etc.) to the real line as long as the mapping is functionally consistent. ${ }^{12}$

The notation $x_{1}, x_{2}$, etc., is also special in that the variables are distinguished with a single index. The domain of the mapping could be multidimensional as with integrals and dot products that map pairs of indices (pairs of functions and pairs of vectors, respectively) to the real line. The mapping is abstract in that it stays unevaluated as part of the analysis. For this reason, such variables are sometimes called uninterpreted functions (Ackermann 1954, Bryant, German and Velev 1999).

\footnotetext{
${ }^{12}$ E.g., $x_{a}$ is the same scalar as $x_{b}$ whenever $a$ is the same as $b$.
} 
Suppose that the integral $\int_{a}^{b} \sin x \ln x d x$ appeared in our model. The uninterpreted function approach is to leave this integral unevaluated, treating $\{\sin x, \ln x, a, b\}$ as the "name" of the scalar variable that is the value of that integral and thereby distinguishing it from, say, $\int_{a}^{b} \cos x \ln x d x$ and $\int_{a}^{c} \sin x \ln x d x$. If our reasoning requires some of the mathematical properties of sin and $\ln$, then the partially interpreted function approach is to add restrictions on the functions, such as $b \geq a \wedge \int_{a}^{b} \sin x \ln x d x \leq(b-a) \ln b \quad$ or $\quad \int_{a}^{c} \sin x \ln x d x=\int_{a}^{b} \sin x \ln x d x+$ $\int_{b}^{c} \sin x \ln x d x$, to the list of assumptions (Kroening and Strichman 2008, p. 73). ${ }^{13}$

\section{III.B. A comparative static in the nonparametric Roy model}

The nonparametric Roy model provides a practical introduction to uninterpreted and partially interpreted functions. In that model, women are assumed to have (possibly correlated) skills $h$ and $r$ in market work and non-market activities, respectively. These skills have a population distribution modeled with the joint density function $f(h, r)$, which is normalized to have unconditional means of zero. Women work if and only if their nonmarket log wage $r+\mu_{r}$ is less than $\sigma h+\mu_{w}$, their market log wage. $\sigma>0$ is a constant introduced for the purposes of considering a comparative static with respect to "wage inequality." The model is "nonparametric" when no specific functional form is assumed for the probability density function.

Applications of the Roy model to the female labor market are abundant in labor supply and econometrics, although often parametric in that $f$ is restricted to be a bivariate normal density function as in the pioneering work of Gronau (1974), Heckman (1979), Heckman and Sedlacek (1985). ${ }^{14}$ Mulligan and Rubinstein (2008) also used the bivariate normal assumption to focus on comparative statics with respect to $\sigma$. Because the bivariate normal density function is not a polynomial in $h$ or $r$, it would seem that the Roy model is not amenable to QE methods. But this overlooks the notion of partially

\footnotetext{
${ }^{13}$ As might be deduced from the previous discussion of QE with free variables, QE can also be used to discover the necessary restrictions on an uninterpreted function.

${ }^{14}$ See also Keane, Moffitt, and Runkle (1988) and Borjas (1994). For some analysis of the nonparametric Roy model, see Heckman and Honore (1990) and Mourifie et al (2017).
} 
interpreted functions, which essentially amounts to a clever choice of variables so that the assumption and hypothesis are understood as Boolean combinations of polynomial inequalities in those variables.

To see this, let's stay with the nonparametric version of the Roy model and look at the definitions of employment $p$ and aggregate market skill $S$ :

$$
\begin{gathered}
p\left(\sigma, \mu_{w}-\mu_{r}\right) \equiv \int_{-\infty}^{\infty} \int_{-\infty}^{\sigma h+\mu_{w}-\mu_{r}} f(h, r) d r d h \\
S\left(\sigma, \mu_{w}-\mu_{r}\right) \equiv \int_{-\infty}^{\infty} \int_{-\infty}^{\sigma h+\mu_{w}-\mu_{r}} h f(h, r) d r d h / p\left(\sigma, \mu_{w}-\mu_{r}\right)
\end{gathered}
$$

$\sigma$ is said to change the "selection rule" if it affects $S$ holding $p$ constant by varying $\mu_{r}{ }^{15}$ With some weak restrictions on the density function, QE confirms that the effect is strictly positive:

$$
\begin{gathered}
A=\left\{\frac{d p\left(\sigma, \mu_{w}-\mu_{r}\right)}{d z}=\frac{d \mu_{w}}{d z}=0 \wedge \frac{d \sigma}{d z}>0 \wedge\right. \\
\int_{-\infty}^{\infty} \int_{-\infty}^{\sigma h+\mu_{w}-\mu_{r}} f(h, r) d r d h \geq 0 \wedge \\
\int_{-\infty}^{\infty} f\left(h, \mu_{w}-\mu_{r}+\sigma h\right) d h>0 \wedge \\
\left.\frac{\int_{-\infty}^{\infty} h^{2} f\left(h, \mu_{w}-\mu_{r}+\sigma h\right) d h}{\int_{-\infty}^{\infty} f\left(h, \mu_{w}-\mu_{r}+\sigma h\right) d h}>\left(\frac{\int_{-\infty}^{\infty} h f\left(h, \mu_{w}-\mu_{r}+\sigma h\right) d h}{\int_{-\infty}^{\infty} f\left(h, \mu_{w}-\mu_{r}+\sigma h\right) d h}\right)^{2}\right\}
\end{gathered}
$$

where any woman with $r=\mu_{w}-\mu_{r}+\sigma h$ is exactly on the margin between work and not work. The first row of assumptions defines the experiment $z$ that increases $\sigma$ and adjusts $\mu_{r}$ to keep employment constant. The second row requires that employment be nonnegative, which reflects the fact that $f$ is a probability density function. The final two

\footnotetext{
${ }^{15}$ It is straightforward to apply QE to questions about the shape of the control function - that is, how $\mu_{r}$ affects $S$ holding $\sigma$ constant. See http://models.economicreasoning.com/SelectionRules.pdf .
} 
rows assume that there are women on the margin and that they are not identical. The hypothesis $H$ is that $\frac{S\left(\sigma, \mu_{w}-\mu_{r}\right)}{d z}>0$.

Using uninterpreted or partially interpreted functions, every atom of $A$ and $H$ above is a polynomial inequality in $\mathbb{R}^{8}$. Treating the total derivative operator as an uninterpreted function, evaluated at points $\left\{\mu_{r}, z\right\},\left\{\mu_{w}, z\right\}$, and $\{\sigma, z\}$, gives us three variables. Treating the single-integral operator as an uninterpreted function, evaluated at points $\left\{1, f\left(h, \mu_{w}-\mu_{r}+\sigma h\right)\right\},\left\{h, f\left(h, \mu_{w}-\mu_{r}+\sigma h\right)\right\}$, and $\left\{h^{2}, f\left(h, \mu_{w}-\mu_{r}+\sigma h\right)\right\}$, gives us three more variables. ${ }^{16}$ The final two variables come from treating the doubleintegral operator as an uninterpreted function evaluated at points $\{1, f(h, r)\}$ and $\{h, f(h, r)\}$. These three functions become "partially interpreted" when we introduce algebraic assumptions about them, as in each atom of the assumptions $A$. It is worth noting at this point that the restrictions that $A$ puts on the probability density function $f$ are satisfied by the Gaussian joint density function, so that any hypothesis deduced from A can also be deduced from the stronger assumption that $f$ is Gaussian.

In summary, the wage-inequality comparative static of the nonparameteric Roy model is a QE problem in eight variables:

$$
\begin{gathered}
\left\{\frac{d \mu_{r}}{d z}, \frac{d \mu_{w}}{d z}, \frac{d \sigma}{d z}, \int_{-\infty}^{\infty} f\left(h, \mu_{w}-\mu_{r}+\sigma h\right) d h,\right. \\
\int_{-\infty}^{\infty} h f\left(h, \mu_{w}-\mu_{r}+\sigma h\right) d h, \int_{-\infty}^{\infty} h^{2} f\left(h, \mu_{w}-\mu_{r}+\sigma h\right) d h \\
\left.\int_{-\infty}^{\infty} \int_{-\infty}^{\sigma h+\mu_{w}-\mu_{r}} f(h, r) d r d h, \int_{-\infty}^{\infty} \int_{-\infty}^{\sigma h+\mu_{w}-\mu_{r}} h f(h, r) d r d h\right\}
\end{gathered}
$$

The result is confirmed by existentially quantifying each of these variables in the counterexample Tarski formula $A \wedge \neg H$, with $A$ and $H$ defined as above, and then eliminating the eight quantifiers to find False: there is no way to assign eight real numbers to those eight scalar variables that would involve simultaneously satisfying the assumptions and contradicting the hypothesis. The role of partially interpreted functions can be seen by comparing the assumptions $A$ expressed in econometrically-natural

\footnotetext{
${ }^{16}$ Recall that the domain of an uninterpreted function does not have to be real numbers. It can be, for example, pairs of integrable functions of $h$ such as $h^{2}$ or $f\left(h, \mu_{w}-\mu_{r}+\sigma h\right)$.
} 
notation as in (10) above with the same assumptions expressed in more generic notation as in (12) below:

$$
A=\left\{x_{2} x_{4}+x_{3} x_{5}-x_{1} x_{4}=x_{2}=0 \wedge x_{3}>0 \wedge x_{7}>0 \wedge x_{4}>0 \wedge \frac{x_{6}}{x_{4}}>\left(\frac{x_{5}}{x_{4}}\right)^{2}\right\}
$$

The hypothesis $H$, derived using the chain rule of calculus, is $x_{1} x_{4} x_{8}+x_{2} x_{5} x_{7}+$ $x_{3} x_{6} x_{7}>x_{1} x_{5} x_{7}+x_{2} x_{4} x_{8}+x_{3} x_{5} x_{8}$. Section $\mathrm{V}$ below shows how software is already available to automatically recognize partially interpreted functions, make the translation from (10) to (12), and then eliminate the quantifiers.

\section{III.C. Omitted variable bias in the linear regression model}

Take the linear regression model (13) with two regressors $v_{1}$ and $v_{2}$ :

$$
y=v_{1} \beta_{1}+v_{2} \beta_{2}+\varepsilon
$$

where $y, v_{1}, v_{2}$, and $\varepsilon$ are each mean-zero vectors of the same length equal to the number of observations, which can be any integer greater than two. I also assume that none of the vectors has any imaginary elements, which is the implicit assumption in essentially all regression analysis but below we see how it matters for QE.

Here we use QE to check whether a zero correlation between $v_{1}$ and $\varepsilon\left(v_{1} \cdot \varepsilon=0\right)$, and an imperfect correlation between the two regressors, guarantees that the OLS regression coefficient on $v_{1}, O L S_{1}$, is equal to $\beta_{1}$. The Tarski formula describing counterexamples is:

$$
\begin{gathered}
v_{1} \cdot \varepsilon=0 \wedge\left(v_{1} \cdot v_{1}\right)\left(v_{2} \cdot v_{2}\right)-\left(v_{1} \cdot v_{2}\right)^{2} \neq 0 \wedge G\left(v_{1}, v_{2}, \varepsilon\right) \wedge \\
\frac{v_{1} \cdot\left(v_{1} \beta_{1}+v_{2} \beta_{2}+\varepsilon\right)\left(v_{2} \cdot v_{2}\right)-\left(v_{1} \cdot v_{2}\right) v_{2} \cdot\left(v_{1} \beta_{1}+v_{2} \beta_{2}+\varepsilon\right)}{\left(v_{1} \cdot v_{1}\right)\left(v_{2} \cdot v_{2}\right)-\left(v_{1} \cdot v_{2}\right)^{2}} \neq \beta_{1}
\end{gathered}
$$


where the ratio term in the formula is $O L S_{1}$ and $G$ is a set of additional assumptions discussed below.

Rather than assigning variable names to every element of all three vectors and then evaluating each of the vector dot products, I treat the dot product as a partially interpreted function. So the counterexample decision problem is whether there exists six real numbers for $\left\{v_{1} \cdot v_{1}, v_{1} \cdot v_{2}, v_{1} \cdot \varepsilon, v_{2} . v_{2}, v_{2} \cdot \varepsilon, \varepsilon . \varepsilon\right\}$ that satisfy (14). ${ }^{17}$ However, given that all three vectors are real valued, some values for these dot products must be ruled out. For example, $v_{1} \cdot v_{1}$ cannot be negative. More specifically, any four real-valued vectors must have a Gramian matrix that is symmetric and positive semi-definite, and with any symmetric positive semi-definite Gramian matrix four real-vectors can be found to assemble that Gramian matrix, as long as the length of the vectors is greater than two. Therefore $G$ must be the conjunction of seven additional assumptions: that each of the three vectors has nonnegative magnitude, that none of the three pairwise correlations is outside the unit circle, and that the determinant of the Gramian matrix is nonnegative. ${ }^{18}$

Quantifying (14)'s six variables existentially and eliminating quantifiers yields True. In words, it is possible to have real-valued data vectors with $v_{1} \cdot \varepsilon=0$ and $\left(v_{1} \cdot v_{1}\right)\left(v_{2} \cdot v_{2}\right)-\left(v_{1} \cdot v_{2}\right)^{2} \neq 0$ but nonetheless $O L S_{1}$ differing from $\beta_{1}$. An additional assumption is needed for $O L S_{1}$ to be unbiased, which QE can recover using much the same procedure that was used in section II.A. Specifically, existential QE is performed on (14) with one free variable, and therefore results in a formula in the free variable. With six variables, there are six ways to do this. ${ }^{19}$ Two of the six results are redundant of the assumptions, which means that these two variables cannot be further restricted to rule out counterexamples. But the other four are not redundant, so we can negate each one to get four conditions that, conditional on the assumptions shown on the top row of (14), are individually sufficient for $O L S_{1}$ to be the same as $\beta_{1}$ :

\footnotetext{
${ }^{17} \beta_{1}$ and $\beta_{2}$ are not variables because they drop out of (14) when the dot products are distributed across addition.

${ }^{18}$ In generic scalar notation, the Tarski formula (14) is $x_{3}=0 \wedge x_{1} x_{4} \neq x_{2}^{2} \wedge\left(x_{1} \geq 0 \wedge x_{4} \geq 0 \wedge\right.$ $\left.x_{6} \geq 0 \wedge x_{1} x_{4} \geq x_{2}^{2} \wedge x_{1} x_{6} \geq x_{3}^{2} \wedge x_{4} x_{6} \geq x_{5}^{2} \wedge 2 x_{2} x_{3} x_{5}+x_{1} x_{4} x_{6} \geq x_{3}^{2} x_{4}+x_{1} x_{5}^{2}+x_{2}^{2} x_{6}\right) \wedge$ $x_{2} x_{5} \neq x_{3} x_{4}$, where the terms in parenthesis are the Gramian matrix restrictions. Gramian matrix restrictions are tedious to type from scratch, but software exists to automatically generate them from the other parts of the Tarski formula (Mulligan, Davenport and England 2018).

${ }^{19}$ In other words, the nonempty set of counterexamples can be projected on to each of six different axes.
} 


$$
v_{2} \cdot \varepsilon=0 \vee v_{1} \cdot v_{2}=0 \vee v_{2} \cdot v_{2}=0 \vee \varepsilon \cdot \varepsilon=0
$$

Note that these results deduced for an econometric model hold for arbitrary-length vectors and therefore can describe either a "sample" or a "population," as long as we are consistent. In other words, the results above by themselves allow us to deduce sample conclusions from sample assumptions or population conclusions from population assumptions. Deriving sample conclusions from population assumptions requires an additional statistical inference step, which is not the subject of these examples. ${ }^{20}$

\section{III.D. Partial identification in the classical measurement error model}

Following Levi (1973), I now add measurement error to one of the two regressors: $\hat{v}_{1}=v_{1}+u .^{21}$ The classical measurement error assumptions are (13):

$$
\begin{gathered}
v_{1} \cdot u=v_{1} \cdot \varepsilon=u \cdot \varepsilon=v_{2} \cdot u=v_{2} \cdot \varepsilon=0 \wedge \\
\beta_{1} \neq 0 \wedge \frac{\left(v_{1} \cdot v_{2}\right)^{2}}{\left(v_{1} \cdot v_{1}\right)\left(v_{2} \cdot v_{2}\right)}<1
\end{gathered}
$$

where I have also added two assumptions to rule out the tedious and less interesting cases that have $\beta_{1}=0$ or perfect collinearity between the $v_{1}$ and $v_{2}$. QE can automatically discover the identified set for the slope parameter $\beta_{1}$ corresponding to the variable $v_{1}$ whose values are measured with error. Here it is helpful to define the forward- and reverse-regression coefficients: ${ }^{22}$

$$
O L S_{1}=
$$

\footnotetext{
${ }^{20}$ See also Franklin Fisher (1966, p. 5) on the distinction between statistical inference and the logical analysis of an econometric model.

${ }^{21}$ See Klepper and Leamer (1984) for analysis of the case where both regressors are measured with error. See Frisch (1934), Friedman (1957), and Tamer (2010) for the single-regressor case.

${ }^{22}$ The reverse-regression coefficient is the inverse of the coefficient on $y$ in the regression of measured $v_{1}$ on $y$ and $v_{2}$.
} 


$$
\begin{gathered}
\frac{\left(v_{1}+u\right) \cdot\left(v_{1} \beta_{1}+v_{2} \beta_{2}+\varepsilon\right)\left(v_{2} \cdot v_{2}\right)-\left(\left(v_{1}+u\right) \cdot v_{2}\right) v_{2} \cdot\left(v_{1} \beta_{1}+v_{2} \beta_{2}+\varepsilon\right)}{\left(\left(v_{1}+u\right) \cdot\left(v_{1}+u\right)\right)\left(v_{2} \cdot v_{2}\right)-\left(\left(v_{1}+u\right) \cdot v_{2}\right)^{2}} \\
R E V_{1}= \\
\frac{\left(v_{1}+u\right) \cdot\left(v_{1} \beta_{1}+v_{2} \beta_{2}+\varepsilon\right)\left(v_{2} \cdot v_{2}\right)-\left(\left(v_{1}+u\right) \cdot v_{2}\right) v_{2} \cdot\left(v_{1} \beta_{1}+v_{2} \beta_{2}+\varepsilon\right)}{\left(\left(v_{1} \beta_{1}+v_{2} \beta_{2}+\varepsilon\right) \cdot\left(v_{1} \beta_{1}+v_{2} \beta_{2}+\varepsilon\right)\right)\left(v_{2} \cdot v_{2}\right)-\left(\left(v_{1} \beta_{1}+v_{2} \beta_{2}+\varepsilon\right) \cdot v_{2}\right)^{2}}
\end{gathered}
$$

The Tarski formula representing the model assumptions - (16), (17), (18) and the 15 restrictions $G\left(v_{1}, v_{2}, \varepsilon, u\right)$ on the Gramian matrix - is a conjunction of polynomial inequalities in 14 scalar variables, which are $\beta_{1}, \beta_{2}, O L S_{1}, R E V_{1}$, and the ten dot products. Existentially quantifying the dot products and $\beta_{2}$, QE results in a formula in the three free variables: ${ }^{23}$

$$
\begin{gathered}
0<O L S_{1} \leq \beta_{1} \leq R E V_{1} \vee \\
R E V_{1} \leq \beta_{1} \leq O L S_{1}<0
\end{gathered}
$$

By construction, (i) the formula (19) must be satisfied in order for the assumptions to be satisfied and (ii) whenever (19) is satisfied there is a way to set the other 11 variables in order to satisfy the assumptions. The first part of the formula (19) is therefore the identified set for $\beta_{1}$ when $\beta_{1}>0$ and the second part corresponds to $\beta_{1}<0$. In words, the identified set is bounded by the forward and reverse regression coefficients. ${ }^{24}$ The identified set is found by projecting the 14-dimensional assumption set into the 3dimensional space $\left\{O L S_{1}, R E V_{1,} \beta_{1}\right\}$. That projection is shown in Figure 2.

\footnotetext{
${ }^{23}$ In generic scalar notation, but for brevity excluding the Gramian matrix restrictions, the Tarski formula representing the assumptions is $x_{6}=x_{7}=x_{9}=x_{10}=x_{12}=0 \wedge \beta \neq$ $0 \wedge x_{4} x_{8} \neq x_{5}^{2} \wedge\left(x_{5}+x_{9}\right)\left(x_{10}+x_{5} \beta\right)=$ for $\left(x_{5}^{2}-x_{11} x_{8}-x_{4} x_{8}-2 x_{6} x_{8}+\right.$ $\left.2 x_{5} x_{9}+x_{9}^{2}\right)+\left[x_{12}+x_{7}+\left(x_{4}+x_{6}\right) \beta\right] x_{8} \wedge \operatorname{rev} x_{10}\left(x_{5}+x_{9}\right)+\left[x_{13}+\beta\left(2 x_{7}+\right.\right.$ $\left.\left.x_{4} \beta\right)\right] x_{8}=x_{10}^{2}+2 x_{10} x_{5} \beta+x_{5}^{2} \beta^{2}+\operatorname{rev}\left\{x_{12} x_{8}+x_{7} x_{8}+\left[\left(x_{4}+x_{6}\right) x_{8}-x_{5} x_{9}-\right.\right.$ $\left.\left.x_{5}^{2}\right] \beta\right\}$.

${ }^{24}$ Below I use a specific QE method to show that the same set (19) is the identified set for some interesting non-classical measurement error models.
} 


\section{III.E. Point identification in discrete choice models}

QE can also be used to study uniqueness or to count numbers of instances. Point identification is a uniqueness question, which for illustration I consider here for the prototypical discrete choice model. The choice is assumed to depend on observed factors $v$ and $y$ as well as unobserved factors summarized by $\sigma \varepsilon$, were $\varepsilon$ is normalized to have unit variance and distribution function $F$. The discrete choice $d_{i}$ is made in situation $i$ if and only if $\sigma \varepsilon_{i}<\beta v_{i}+\gamma y_{i}$. The log likelihood function for observing $v, y, d$ is:

$$
L\left(\left\{\frac{\beta}{\sigma}, \frac{\gamma}{\sigma}\right\}\right)=\sum_{i}\left\{\left(1-d_{i}\right) \ln \left[1-F\left(\frac{\beta}{\sigma} v_{i}+\frac{\gamma}{\sigma} y_{i}\right)\right]+d_{i} \ln F\left(\frac{\beta}{\sigma} v_{i}+\frac{\gamma}{\sigma} y_{i}\right)\right\}
$$

By definition, the parameter vector $\theta \equiv\{\beta, \gamma, \sigma\}$ is point identified if two distinct values for that vector cannot simultaneously attain the maximum likelihood. Conversely, the parameter vector $\{\beta, \gamma, \sigma\}$ is point identified if knowing that $\theta_{1}$ and $\theta_{2}$ both attain the maximum likelihood implies that $\theta_{1}=\theta_{2}$.

The definition of a strictly concave function $L$ is:

$$
\begin{aligned}
{\left[\operatorname{Distinct}\left(\left\{\frac{\beta_{1}}{\sigma_{1}}, \frac{\gamma_{1}}{\sigma_{1}}\right\},\left\{\frac{\beta_{2}}{\sigma_{2}}, \frac{\gamma_{2}}{\sigma_{2}}\right\}\right) \wedge 0<\lambda<1\right] } \\
\Rightarrow\left[(1-\lambda) L\left(\left\{\frac{\beta_{1}}{\sigma_{1}}, \frac{\gamma_{1}}{\sigma_{1}}\right\}\right)+\lambda L\left(\left\{\frac{\beta_{2}}{\sigma_{2}}, \frac{\gamma_{2}}{\sigma_{2}}\right\}\right)\right. \\
\left.<L\left((1-\lambda)\left\{\frac{\beta_{1}}{\sigma_{1}}, \frac{\gamma_{1}}{\sigma_{1}}\right\}+\lambda\left\{\frac{\beta_{2}}{\sigma_{2}}, \frac{\gamma_{2}}{\sigma_{2}}\right\}\right)\right]
\end{aligned}
$$

where Distinct is an uninterpreted Boolean-valued function introduced to compactly represent whether its two arguments are distinct. It can be partially interpreted by providing an algebraic definition of distinct:

$$
\operatorname{Distinct}\left(\left\{\frac{\beta_{1}}{\sigma_{1}}, \frac{\gamma_{1}}{\sigma_{1}}\right\},\left\{\frac{\beta_{2}}{\sigma_{2}}, \frac{\gamma_{2}}{\sigma_{2}}\right\}\right) \Rightarrow\left[\frac{\beta_{1}}{\sigma_{1}} \neq \frac{\beta_{2}}{\sigma_{2}} \vee \frac{\gamma_{1}}{\sigma_{1}} \neq \frac{\gamma_{2}}{\sigma_{2}}\right]
$$

If $\theta_{1}$ and $\theta_{2}$ are both attaining the maximum likelihood, then we have: 


$$
\begin{gathered}
L\left(\left\{\frac{\beta_{1}}{\sigma_{1}}, \frac{\gamma_{1}}{\sigma_{1}}\right\}\right) \geq L\left((1-\lambda)\left\{\frac{\beta_{1}}{\sigma_{1}}, \frac{\gamma_{1}}{\sigma_{1}}\right\}+\lambda\left\{\frac{\beta_{2}}{\sigma_{2}}, \frac{\gamma_{2}}{\sigma_{2}}\right\}\right) \wedge \\
L\left(\left\{\frac{\beta_{2}}{\sigma_{2}}, \frac{\gamma_{2}}{\sigma_{2}}\right\}\right) \geq L\left((1-\lambda)\left\{\frac{\beta_{1}}{\sigma_{1}}, \frac{\gamma_{1}}{\sigma_{1}}\right\}+\lambda\left\{\frac{\beta_{2}}{\sigma_{2}}, \frac{\gamma_{2}}{\sigma_{2}}\right\}\right)
\end{gathered}
$$

Treating the likelihood function $L$ as another partially interpreted function, the 11 scalar and Boolean variables in this model are: ${ }^{25}$

$$
\begin{gathered}
\left\{\text { Distinct }\left(\left\{\frac{\beta_{1}}{\sigma_{1}}, \frac{\gamma_{1}}{\sigma_{1}}\right\},\left\{\frac{\beta_{2}}{\sigma_{2}}, \frac{\gamma_{2}}{\sigma_{2}}\right\}\right), \lambda, \beta_{1}, \beta_{2}, \gamma_{1}, \gamma_{2}, \sigma_{1}, \sigma_{2},\right. \\
\left.L\left(\left\{\frac{\beta_{1}}{\sigma_{1}}, \frac{\gamma_{1}}{\sigma_{1}}\right\}\right), L\left(\left\{\frac{\beta_{2}}{\sigma_{2}}, \frac{\gamma_{2}}{\sigma_{2}}\right\}\right), L\left((1-\lambda)\left\{\frac{\beta_{1}}{\sigma_{1}}, \frac{\gamma_{1}}{\sigma_{1}}\right\}+\lambda\left\{\frac{\beta_{2}}{\sigma_{2}}, \frac{\gamma_{2}}{\sigma_{2}}\right\}\right)\right\}
\end{gathered}
$$

The point-identification question is whether there are values to assign these 11 variables that would simultaneously satisfy (21), (22), (23), $0<\lambda<1, \sigma_{1}>0, \sigma_{2}>0$, and $\beta_{1} \neq \beta_{2} \vee \gamma_{1} \neq \gamma_{2} \vee \sigma_{1} \neq \sigma_{2}$. There are such values, which means that the parameter vector is not point identified. Moreover, QE software typically can provide an example of such values. One example is $\{$ False, $1 / 2,0,0,-2,-1,1,1 / 2,0,0,0\}$, respectively.

QE can also confirm that the parameter ratio $\beta / \gamma$ is point identified. Specifically, QE confirms that there are no values to assign the 11 variables that would simultaneously satisfy (21), (22), (23), $0<\lambda<1, \sigma_{1}>0, \sigma_{2}>0$, and $\beta_{1} / \gamma_{1} \neq \beta_{2} / \gamma_{2}{ }^{26}$

The model (21), (22), and (23) is quite simple because concavity of the likelihood function is assumed directly, rather than derived as a result based on other assumptions. But the example's purpose is just to illustrate that uniqueness questions, and therefore point-identification questions, fit in the Tarski framework merely by querying whether

\footnotetext{
${ }^{25}$ The QE framework is for scalar variables but note that a Boolean variable can be trivially converted in to a scalar variable by creating a new scalar variable $z$ and replacing all instances of the Boolean variable with $z>0$.

${ }^{26}$ In generic scalar notation, the Tarski formula for the assumptions is $x_{2} \geq x_{3} \wedge x_{4} \geq x_{3} \wedge 0<$ $x_{1}<1 \wedge x_{10}>0 \wedge x_{11}>0 \wedge\left[x_{1} x_{2}+x_{3}>x_{1} x_{4}+x_{2} \vee\left(x_{7} x_{10}=x_{6} x_{11} \wedge x_{8} x_{11}=x_{9} x_{10}\right)\right]$.
} 
two potentially distinct points satisfying the model assumptions must coincide with each other.

\section{III.F. A Library of Economics Examples}

Since 2016 I have been using automated QE on a daily basis to process problems encountered in teaching, research, and writing a graduate-level textbook. The more interesting or well known of these problems have been assembled together with background explanations at http://examples.economicreasoning.com. Mulligan et al (2018a) provide the computer science community with forty-five of these assumptionhypothesis pairs (for a total of 135 existential sentences), in four different computer algebra formats, together with analysis of their algebraic structure and explanation of the range of economics problems that fit in the QE framework.

The examples shown in this paper, chosen to illustrate QE concepts, are more stylized than in the library of 45. Even excluding the two-state example (5) - (7), this paper's examples have an average of only 9.25 variables as compared to 17.2 in the library. ${ }^{27}$ This suggests that significantly more involved econometrics models could still be decided in seconds, as the library examples are (see also Table 2).

\section{Relevant Theorems from Real Algebraic Geometry}

\section{IV.A. Tarski: Real quantifier elimination is always possible \\ Mathematician and logician Alfred Tarski proved that there exists a universal} algorithm (that is, one not requiring problem-specific guidance) for quantifier elimination from systems of polynomial inequalities on real closed fields by providing such an algorithm. ${ }^{28}$ Because the real numbers are an example of a real closed field, the Tarski result guarantees that there exists a quantifier-free formula $P$ satisfying (1) and (4) and

\footnotetext{
${ }^{27}$ The library has a greater incidence of polynomials containing a cubic in a single variable, although the classical measurement error model's hypothesis does have a single variable raised to the fourth power.

${ }^{28}$ Tarksi made the proof in 1930 (Caviness and Johnson 1998, p. 1), but the result was not published until Tarski (1951).
} 
gives an algorithm for finding $P$. If $Q R$ is a sentence, then the $\mathrm{QE}$ algorithm is a "decision method": a procedure for determining whether $Q R$ is True or False. ${ }^{29}$

\section{IV.B. Collins: A more efficient algorithm for real QE that defines sets recursively}

Although Tarski's method is enough to prove that quantifiers can be eliminated, it is not used in practice due to its "extreme" inefficiency. ${ }^{30}$ A major step forward came with the Cylindrical Algebraic Decomposition (CAD) method introduced by mathematician George E. Collins in $1973 .{ }^{31}$

\section{IV.B.1. Properties of CAD}

In our setting (1) and (4), the CAD method decomposes $\mathbb{R}^{N}$ into finitely many connected regions, known as "cells," with three properties:

(i) each cell of the CAD is a semi-algebraic set (i.e., it is defined by a finite number of quantifier-free polynomial inequalities).

(ii) The CAD result is cylindrical because the projections of any two of the cells into $\mathbb{R}^{k}, 1 \leq k \leq N$, are either identical or disjoint.

(iii) Each cell is adapted to the Tarski formula from which it was derived, which means that none of the polynomials in the Tarski formula $T$ has more than one sign $\{-1,0,1\}$ in any one of the cells.

Every Tarski formula has such a CAD (Basu, Pollack and Roy (2011, Theorem 5.6)).

The T-adapted (i.e., uniform sign) property of the cells, and the fact that the cells are finite in number, means that any quantified formula can be confirmed in a finite number of steps. ${ }^{32}$ The cylindrical property (in economics we would call it "recursive") of the decomposition means that the cells have a natural ordering and many times can be processed more than one at a time.

\footnotetext{
${ }^{29}$ Renegar (1998, p. 221).

${ }^{30}$ Arai, et al. (2014). See also Davenport, Siret and Tournier (1988, p. 119), who describe Tarski's method as "completely impractical."

${ }^{31}$ Collins (1973) and Collins (1975).

${ }^{32}$ By construction, the Tarski formula is True at any one point in a cell if and only if it is True everywhere in that cell.
} 
The phrase CAD has a number of related but distinct uses in mathematics and computer science. Narrowly speaking, CAD refers to a method, or sometimes an expanded set of polynomials (including, among others, those in the original Tarski formula) obtained by the method, or the full collection of cells obtained by the method. Another result of the CAD method is its cells, described by Cylindrical Algebraic Formulas (CAFs), which may also be referenced as CAD. ${ }^{33}$ CAD sometimes also refers to a decomposition of part of $\mathbb{R}^{N}$ with the properties (i)-(iii). CAD can also refer to a full decomposition of $\mathbb{R}^{N}$ with the properties (i)-(iii), but with "adapted" defined with respect to the truth value of the entire formula rather than the sign of each of its polynomials (Bradford, et al. 2016). For clarity, I refer to the full collection of cells together with one sample point each as "the full CAD."

Although building the CAD begins with a Tarski formula, only the polynomials of that formula and the order of quantification is used in the calculations (recall (iii)); the inequalities and Boolean operations are ignored. A single full CAD therefore solves a large number of QE problems: any $\mathrm{QE}$ problem whose Tarski formula has the same polynomials and the same quantification order (but not necessarily the same quantifiers) has the same full CAD. Naturally, the simultaneous solution of many QE problems requires more computational resources than solving one QE problem, which is the motivation for other QE algorithms.

Kauers (2011) and Mulligan (2016) include explanations, intended for nonexperts, of the methods used to construct a full CAD. Given that the full CAD would rarely be the best $\mathrm{QE}$ method for econometrics problems and that it is already implemented in various software packages, the details of its construction is beyond the scope of this paper.

\section{IV.B.2. Using CAFs in Econometrics: Necessary and Sufficient Conditions in the Measurement Error Model}

Recall the measurement error model whose assumptions - (16), (17), (18) and the 15 restrictions $G\left(v_{1}, v_{2}, \varepsilon, u\right)$ - guarantee that the regression parameter $\beta_{1}$ is in the set

\footnotetext{
${ }^{33}$ See Strzebonski (2010) and Chen and Maza (2015) for more on the distinction between CAD and $\mathrm{CAF}$.
} 
(19) bounded by the forward and reverse regression coefficients. Here I consider an interesting case of non-classical measurement error by weakening the first restriction to $v_{1} . u \geq 0$. In words, the error in measuring $v_{1}$ can be positively correlated with $v_{1}$. Now the set of counterexamples (cases with $\beta_{1}$ outside of the set (19)) would not be empty, and we could project that set onto each of the 14 axes to find sufficient conditions for the set (19) to continue to be the identified set. Two are found:

$$
v_{1} \cdot u=0 \vee u \cdot u=0
$$

Each of (25) is a sufficient condition because it was obtained with projection onto an axis, which shows values of that variable that are inconsistent with counterexamples but maybe not required for an example. In other words, examples are described by the set $A$ $\wedge H$, and sufficient conditions are potentially inconsistent with parts of this set.

Admittedly (25) is not an interesting addition to the classical measurement error model, but it is too soon to conclude that the classical model is the only interesting case having (19) as its identified set. Perhaps there are interesting examples in $A \wedge H$ that are ruled out by the sufficient conditions (25). The answer is found by looking at the weaker necessary and sufficient conditions. CAD is helpful here because it can provide a relatively simple, but not tautological, formula for $A \wedge H$. The CAD depends on quantification order, of which there are 14 ! possibilities in this example. Some of these are simpler than others. Others just show the hypothesis formula. But some of them may be useful (and software is available to help find the useful one).

One CAF expression for the necessary and sufficient conditions for the weakened measurement error problem is (26):

$$
\beta_{1} \neq 0 \wedge v_{1} \cdot u \geq 0 \wedge \varepsilon \cdot \varepsilon \geq \beta_{1}^{2} v_{1} \cdot u
$$


Both (25) and (26) rule out all counterexamples, but the latter is the weaker condition because it does not rule out any cases satisfying $A \wedge H .^{34}$ Notice the CAF's recursive structure: it first restricts $\beta_{1}$ relative to real numbers only, then restricts the second variable $v_{1} \cdot u$ based on the first (trivially in this case), and then restricts the third variable $\varepsilon . \varepsilon$ based on the first two. In terms of the econometric substance, (26) shows that (19) is still the identified set even when the measurement error is positively correlated with the true value, as long as that correlation is not too positive.

\section{IV.C. Other QE methods}

Basu, Pollack and Roy (2011) have a unique approach to QE problems, although not yet implemented as software. Less ambitious and computationally less costly (than full CAD) algorithms are available for special cases of the QE problem. Virtual Term Substitution (VTS) is designed for QE on polynomials with low own degree: that is, the total degree may be large because several variables may multiply each other, but it is rare for a single variable to be raised to a power of more than two or three. ${ }^{35}$ The performance of VTS has an additional advantage in large but sparse systems where most variables are absent from most of the polynomials in the Tarski formula. VTS is therefore well-suited for problems in econometrics and elsewhere in economics.

\footnotetext{
${ }^{34}$ An example that satisfies $A$ and (26) without satisfying (25): $\beta_{1}=v_{1} \cdot v_{1}=v_{1} \cdot u=v_{2} \cdot v_{2}=$ $u . u=\varepsilon \cdot \varepsilon=1 \wedge v_{1} \cdot v_{2}=v_{1} \cdot \varepsilon=v_{2} \cdot u=v_{2} \cdot \varepsilon=u \cdot \varepsilon=0$.

${ }^{35}$ VTS was invented by Volker Weispfenning $(1988,1997)$. Improvements to the method are ongoing, as with C.W. Brown (2005).
} 
Regardless of degree, decision problems are a special case of QE for which algorithms can be tailored. The decision of existential sentences has received much attention in computer science, and specialized methods have been implemented by a number of automated SMT solvers with NRA capabilities. ${ }^{36}$ The aforementioned library of economic decision problems are special not only in degree, but also that the process of repeated quantifier elimination involves just a few frequently repeated single-variable quantifier elimination problems. Mulligan et al (2018b) show how pattern recognition can quickly reach decisions in these cases without relying on any other QE method, even while the same satisfiability problems are not decided with current SMT-NRA solvers.

\section{IV.D. The Computational Complexity of QE}

The worst-case complexity of (that is, the computational resources theoretically needed for) QE with a single type of quantifier is asymptotically exponential in the number of variables (Grigor'ev 1988, Basu, Pollack and Roy 2011). However, singleexponential QE methods have not yet been implemented as software (Davenport and England 2015, Sturm 2017). The construction of a full CAD has worst-case complexity that is asymptotically double-exponential in the number of variables, with the base of those exponents proportional to the product of the number of polynomials and their average degree, even if the formula contains only linear polynomials (Brown and Davenport 2007, England and Davenport 2016).

QE algorithms have rarely been discussed in economics, but in these few cases appear notorious for the full CAD's theoretical asymptotic properties. In his lectures to Yale economics professors, mathematician Charles Steinhorn (2008, p. 177) conjectured that "... quantifier elimination is something that is do-able in principle, but not by any computer that you and I are ever likely to see. ${ }^{37}$ In their discussion of automating highschool level mathematics, Arai, et al. (2014p. 7) warned that “... the calculation time

\footnotetext{
${ }^{36}$ See Jovanović and de Moura (2012) for an exposition of SMT-NRA methods.

${ }^{37}$ Although Steinhorn added "Well, I'll retract that last statement because it's probably false." See also Carvajal, et al. (2014, p. 260) who wrote in Economic Theory that the CAD algorithm to "implement this elimination of quantified variables ... [is] known to be doubly exponential."
} 
required for $\mathrm{CAD}$ is doubly exponential in the number of variables $n$ in the proposition supplied. The practical limit to obtain a solution would be at most five variables."

The dismissal of QE algorithms in economics has been based on theoretical asymptotic complexity results rather than experience with actual software applied to actual economic reasoning. If we expect that an algebraic deduction problem could be solved manually in reasonable time, why wouldn't a machine be able to solve it in seconds? In practice, seconds is all that it takes for QE software to solve many meaningful problems in econometrics, and in economics generally.

The discrepancy between reputation and practice comes from a combination of:

(i) the approximation error in asymptotic complexity theory,

(ii) the algebraic structure of the applied problems, and

(iii) the distinction between a full CAD and alternative $\mathrm{QE}$ methods that use the Boolean and relational structures of each problem.

The potential complexity of the full $\mathrm{CAD}$, which ignores everything about the Tarski formula except for the $M$ polynomials it contains (after factoring), comes from the $\operatorname{Binomial}_{2}(M)$ number of ways that $M$ polynomials can intersect pairwise because each intersection is at the border of polynomial sign changes. Eliminating a single variable from a formula with $M$ polynomials therefore results in up to $\operatorname{Binomial}_{2}(M)$ polynomials to be examined in the next stage. ${ }^{38}$ After eliminating a second variable, there will be up to $\operatorname{Binomial}_{2}\left(\operatorname{Binomial}_{2}(M)\right)$ polynomials. Eliminating $N$ variables this way nests the Binomial $_{2}$ function $N$ times.

The leading term in the $N$-times-nested Binomial $_{2}$ function is $2(M / 2)^{2^{N}}$, which is doubly exponential and can grow quite rapidly with $N$. But note that the leading term can be a poor approximation of the nested binomial, especially when $M<4$, in that it exaggerates the magnitude of the growth rate of the number of polynomials and for $M \leq 3$

\footnotetext{
${ }^{38}$ This discussion ignores the polynomials that must also be introduced to track singularities, but these do not grow (with successive variable eliminations) at the rate that intersections can. See C.W. Brown (2001) for further discussion. Moreover, in practice, the polynomials representing singularities, such as variable sign conditions, are often already part of the original Tarski formula.
} 
even gets the sign wrong. ${ }^{39}$ A significant number of economic examples have its average variable appearing in no more than three polynomials and therefore full CADs can be constructed without difficulty even while each example has ten or more variables.

At the same time, a number of examples in econometrics and elsewhere in economics are too algebraically complicated for full CAD construction to be practical. Nevertheless QE is practical, and often achieved in mere milliseconds, with QE methods that are tailored to the Boolean and algebraic structure of the problem. ${ }^{40}$ Table 2 shows the decision times for the four examples in this paper (excluding the two-state model), as well as a summary of the decision times for the library of 45 examples from economic theory. The three software implementations used, as well as others, are discussed next.

\section{QE Software}

There are modern software implementations of QE in Mathematica (Strzebonski 2010, 2016), REDLOG (Dolzmann and Sturm 1997), Maple (Anai and Yanami 2003, Chen and Maza 2016) and QEPCAD-B (C. W. Brown 2003). In principle, these tools can solve any QE problem in finite time, given enough computing resources. However, some user input, such as the order for eliminating variables, is recommended so that the QE algorithm runs efficiently on the problem at hand. REDLOG and QEPCAD-B are free software.

QE for existential sentences is also soluble using the technology of Satisfiability Modulo Theory (SMT) Solvers; at least those that support the QF_NRA logic such as SMT-RAT (Corzilius, et al. 2012), veriT (Fontaine, et al. 2017), Yices2 (Jovanović and Dutertre 2017), and Z3 (Jovanović and de Moura 2012). These do not guarantee a decision: the software authors note that it is possible that the software returns "unknown" or enters an infinite loop. These SMT solvers are free.

\footnotetext{
${ }^{39}$ For $M=3$, the leading term is 83 billion times larger for the elimination of the sixth quantifier than it is for eliminating the first, whereas $\operatorname{Binomial}_{2}(M)$ nested six times is no different from Binomial $_{2}(3)$ itself.

${ }^{40}$ See also Shankar (2002, p. 13), who explains that "[m]any decision procedures are of exponential, super-exponential, or non-elementary complexity. However, this complexity often does not manifest itself on practical examples."
} 
I created a Mathematica package, intended to sharply lower the cost to economists of using the QE tools in Mathematica, REDLOG, and SMT-NRA solvers. The user inputs only assumptions and hypothesis, in a natural format much like shown in (10). The software then automatically: checks for errors; parses and standardizes input; assembles the Tarski formula using partially interpreted functions as needed; adds Gramian-matrix assumptions as needed; makes algorithm choices and passes the QE problem to a QE engine; interprets the QE results according to Table 1; and suggests what the user can do next. The QE engine is primarily Mathematica's RESOLVE function, but the package is also capable of writing code for REDLOG and SMT-NRA (especially, Z3). Figure 3 is a Mathematica screen shot showing the processing of the comparative static for the nonparametric Roy model; the technical details of QE are invisible to the user.

The free package is available by evaluating Get["http://economicreasoning.com"] at a Mathematica prompt. Further information about Economicreasoning use and technical background are available from http://help.economicreasoning.com and Mulligan, Davenport, and England (2018).

With these software resources available, automated QE is easy to perform: about as easy as running a regression with a modern statistics package. When called by the Economicreasoning package in deduction problems encountered in my economics teaching, research, and writing a graduate-level textbook, Mathematica's algorithm always performs the QE in seconds. ${ }^{41}$ At least one of the problems automatically solved in seconds is familiar from macro and public economics yet experts in the field have been known to get it wrong when they attempt it manually. ${ }^{42}$ REDLOG can do most of the same QE problems even faster, although Mathematica (and Z3) more often accelerates computation by (appropriately) discarding irrelevant parts of a formula. Z3 tends to be even faster than REDLOG with the QE problems it can solve, which is a large majority of the ones that I have tried, but also fails to solve a few percent of the problems. Like other SMT solvers, Z3 cannot perform QE with free variables.

\footnotetext{
${ }^{41}$ This is not to say that Mathematica's QE is without limits, just that my practical and frequent usage has not yet tested those limits.

${ }^{42}$ See the Laffer curve problem in Mulligan (2016). Economicreasoning has also been used to generate novel and substantive conclusions about the economy (Mulligan and Tsui 2016).
} 


\section{Conclusions}

If-then statements about statistics, econometric models, etc., dating back to the early pioneers of formal statistical reasoning, are implicit eliminations of "for all" quantifiers from a True sentence. This paper makes the quantifier elimination (QE) explicit and thereby brings to bear applicable and relatively new tools from real algebraic geometry and computer science.

QE algorithms automatically decide the truth of hypotheses in finite time, without approximation or functional-form assumptions. The algorithms can thereby also help formulate and understand hypotheses by detecting inconsistent assumptions, calculating sufficient conditions, calculating necessary and sufficient conditions, and generating examples. These results are not merely hopeful conjectures for the practice of econometrics. Software is already available for automatically eliminating quantifiers, which I have incorporated into an economist-friendly interface running in Mathematica. All of the hypotheses in this paper were refuted or verified merely by entering them as assumptions and potential implications, as shown in Figure 3.

The QE framework requires statements that are, interpreted in the right space, quantified ("for all") Tarski formulas, each of which is a quantifier-free Boolean combination of polynomial inequalities. In order for an econometric hypothesis to fit in this framework, its elements need not be polynomial functions: the hypothesis just has to be stated in terms of properties of the model that are expressed as a finite number of polynomial relationships among real numbers.

A wide range of econometric models can be processed in this way, although more work is needed to expand the range, and better understand the practical limits, of hypotheses and proofs that can be automated with quantifier elimination. QE methods cannot be applied until the polynomial structure of the problem is discovered. Existing software is often capable of automatically discovering that structure, but in other cases the software user needs to assist in the discovery by consciously using partially interpreted functions rather than specific functional forms. Presumably partially interpreted functions are more natural to econometricians working with nonparametric models than those more familiar with parametric approaches. 
Because QE is automatically done by software, the details of implementation are of limited interest to users. However, a few implementation concepts are helpful. The Cylindrical Algebraic Decomposition (CAD) approach defines sets with recursive formulas, which can clearly delineate cases of interest to the user and sometimes provide a practical roadmap for step-by-step proofs (Mulligan 2016). The CAD also neatly illustrates how "ForAll" statements about $\mathbb{R}^{N}$ can be rigorously confirmed by considering just a finite number of examples. But full CAD construction, which receives relatively more attention in the theoretical asymptotic complexity literature, is usually doing far more than is necessary to perform $\mathrm{QE}$ on a particular problem. ${ }^{43}$ Most software implementations are therefore not constructing a full CAD.

Two quite distinct types of methods are used in modern QE software. One type refines $\mathrm{CAD}$ to take advantage of the special algebraic structure of a problem, which is to be expected for problems that people might solve "manually." The second type consists of nonlinear arithmetic extensions to algorithms for solving the satisfiability in computer science, some of which are used commercially for certifying the performance of computer hardware and software. The first type can, with enough computation resources, solve any QE problem. The second type is limited to deciding existential sentences, which can be a workhorse for deduction in econometric theory. Both types of methods continue to be advanced and adapted to practical problems, now with the advantage of substantively interesting examples from economics and statistics (Mulligan, Bradford, et al. 2018a).

The QE methods in this paper deliver certified conclusions, but a conclusion is not the same as a concise proof. The internal software steps of QE are themselves a proof, and some of the QE software is capable of displaying or summarizing them, but usually the software steps are too lengthy and tedious for a human reader to appreciate or practically verify. But even in those cases QE could be of tremendous assistance to someone attempting to construct a concise proof by: confirming that a hypothesis is provable, investigating the equivalence of one hypothesis with another, incrementally eliminating or modifying assumptions to see which of them are binding, verifying any

\footnotetext{
${ }^{43}$ Moreover, for many practical problems, the asymptotic approximations said to describe full CAD construction not only produce the wrong orders of magnitude, but also the wrong sign.
} 
number of intermediate results that may serve as one of the steps in the proof, and automatically generating examples.

Human error can result in logically or mathematically erroneous conclusions, whether those conclusions were generated with a machine or with pencil and paper. In the latter approach, a diligent reader, editor, or referee, also operating with pencil and paper, has been required to detect and correct publication errors. Human errors could in principle be embedded in QE software (Davenport \& England, 2015), although QE methods can decide any universal sentence with two different methods (i.e., the left- and right-hand sides of (3) potentially involve different software steps) and each of those decisions can be processed in $N$ ! different sequences ( $N$ is the number of quantified variables). Moreover, multiple software packages are available to perform the same calculation (this paper uses Mathematica, REDLOG, and Z3), not to mention the fact that the owners of commercial software packages have both the opportunity and incentive to find and correct software errors. ${ }^{44}$ Also note that empirical economics publications already include dozens, if not hundreds, of matrix inversions that are never verified with pencil and paper or even with an alternative software package. Perhaps econometric theory will follow a similar path.

\footnotetext{
${ }^{44}$ If the machine-generated conclusion is that either an example or counter example exists, then this can readily be verified with pencil and paper because the software provides the example.
} 
Table 1. Possible Outcomes from a Potential Theorem $\forall v\left[A_{v} \Rightarrow H_{v}\right]$

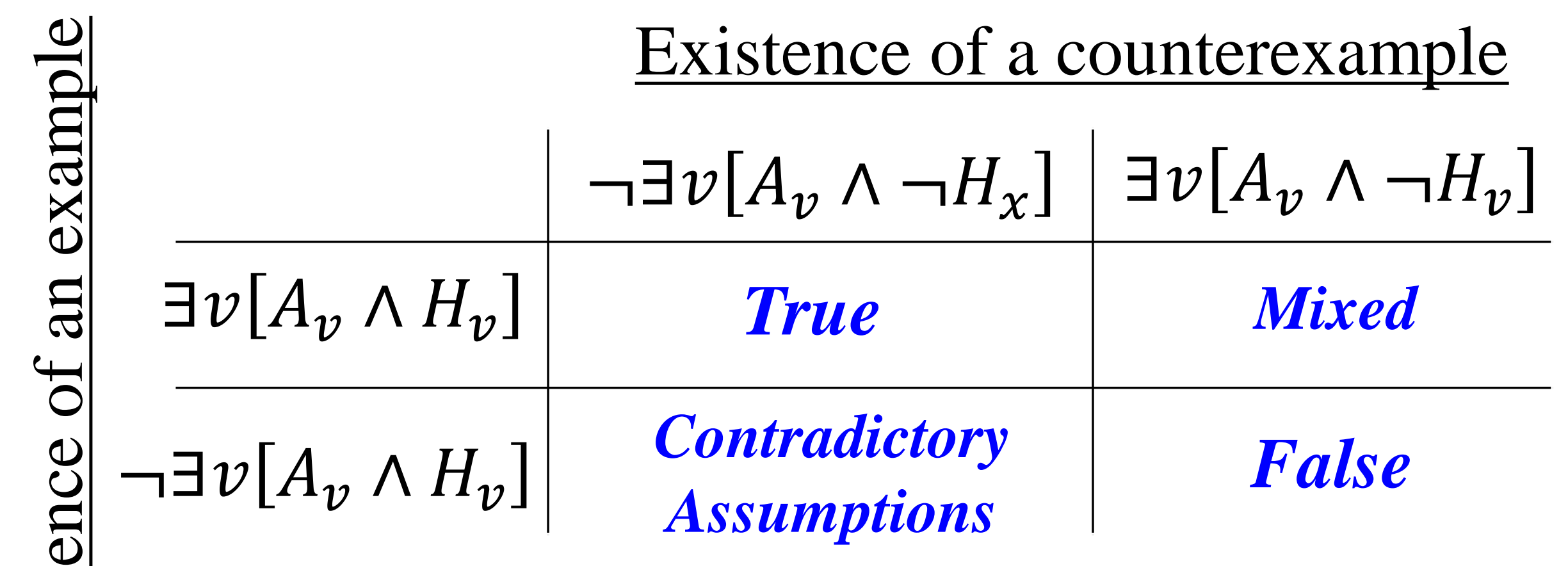

$A_{v}$ and $H_{v}$ are each a Tarski formula in the variables $v$. "Assumption" and "Hypothesis" 
Table 2. Decision times for the econometrics examples

Times for deciding universal sentences, in milliseconds

\begin{tabular}{|c|c|c|c|c|}
\hline \multirow[b]{2}{*}{ Model } & \multirow{2}{*}{$\begin{array}{l}\text { Dimensions } \\
\text { represented }\end{array}$} & \multicolumn{3}{|c|}{ Decision time (milliseconds) } \\
\hline & & Mathematica & REDLOG & $\mathrm{Z3}$ \\
\hline \multicolumn{5}{|l|}{ The Identified Set in the Classical } \\
\hline Measurement Error Model & 12 & 2,010 & 70 & 20 \\
\hline Nonparametric Roy Model & 8 & 773 & $<50$ & $<15$ \\
\hline Omitted Variable Bias & 6 & 62 & $<50$ & $<15$ \\
\hline \multicolumn{5}{|l|}{ Parameter Identification in } \\
\hline Discrete-choice Models & 11 & 85 & $<50$ & $<15$ \\
\hline \multicolumn{5}{|l|}{ Addendum: } \\
\hline Medians from 45-example library & 14 & 825 & 50 & $<15$ \\
\hline
\end{tabular}

Note : Universal sentences state a hypotheses to be True for all $N$-dimensional real numbers, where $N$ is the number of dimensions needed to represent the model. Computer time was calculated with Mathematica 11.2, the PSL version of REDLOG (revision 4330), and version 4.5.0 of Z3 on a Macbook Pro Mid 2014 2.8GHz Interl i7. REDLOG (Z3) failed to decide one (two) from the 45example library, respectively. 


\section{Figure 1. The Set of Counterexamples}

The Two-State Model

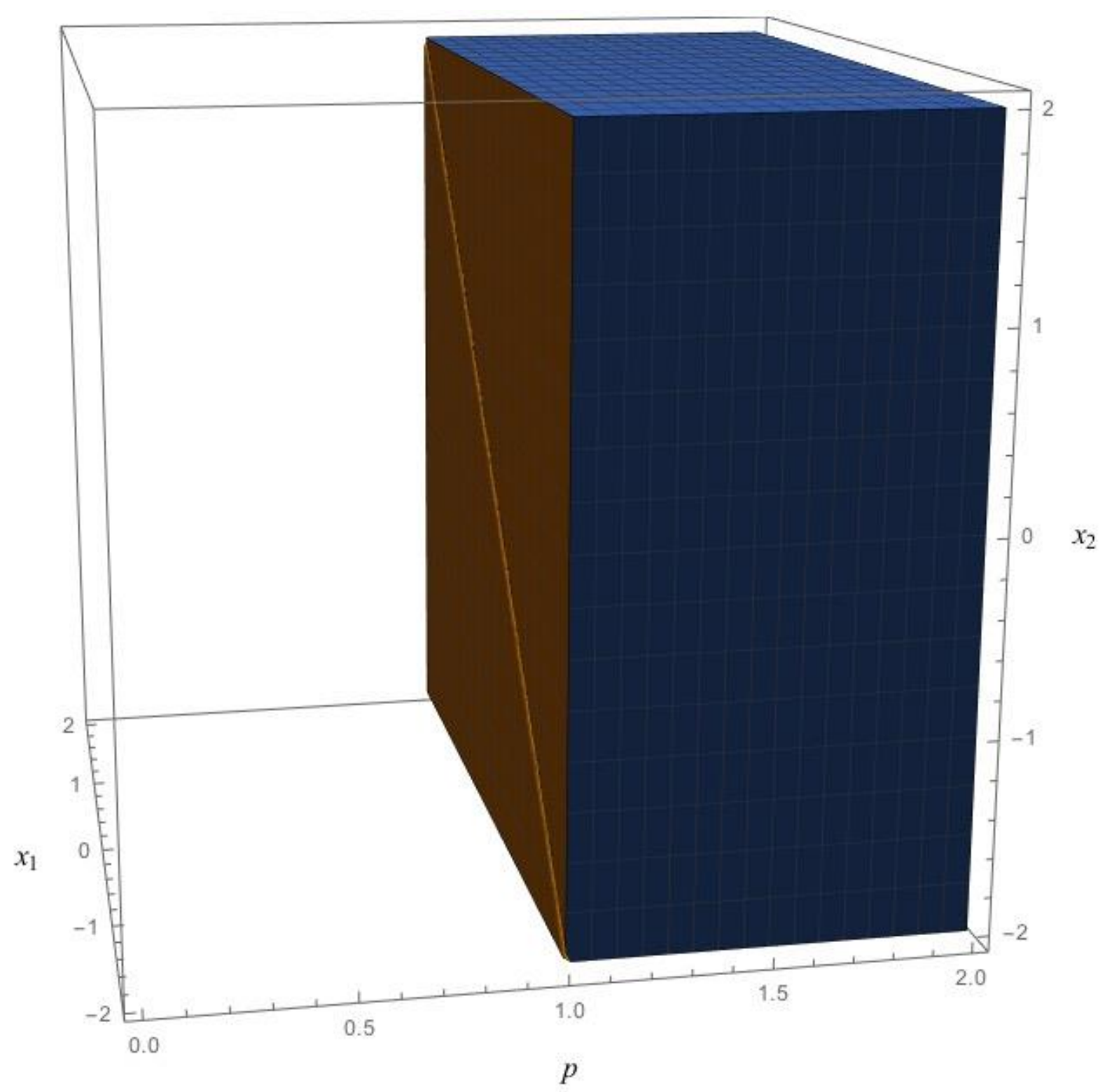


Figure 2. The Identified Set in the Classical Measurement Error Model

14-dimensional assumptions projected down to three

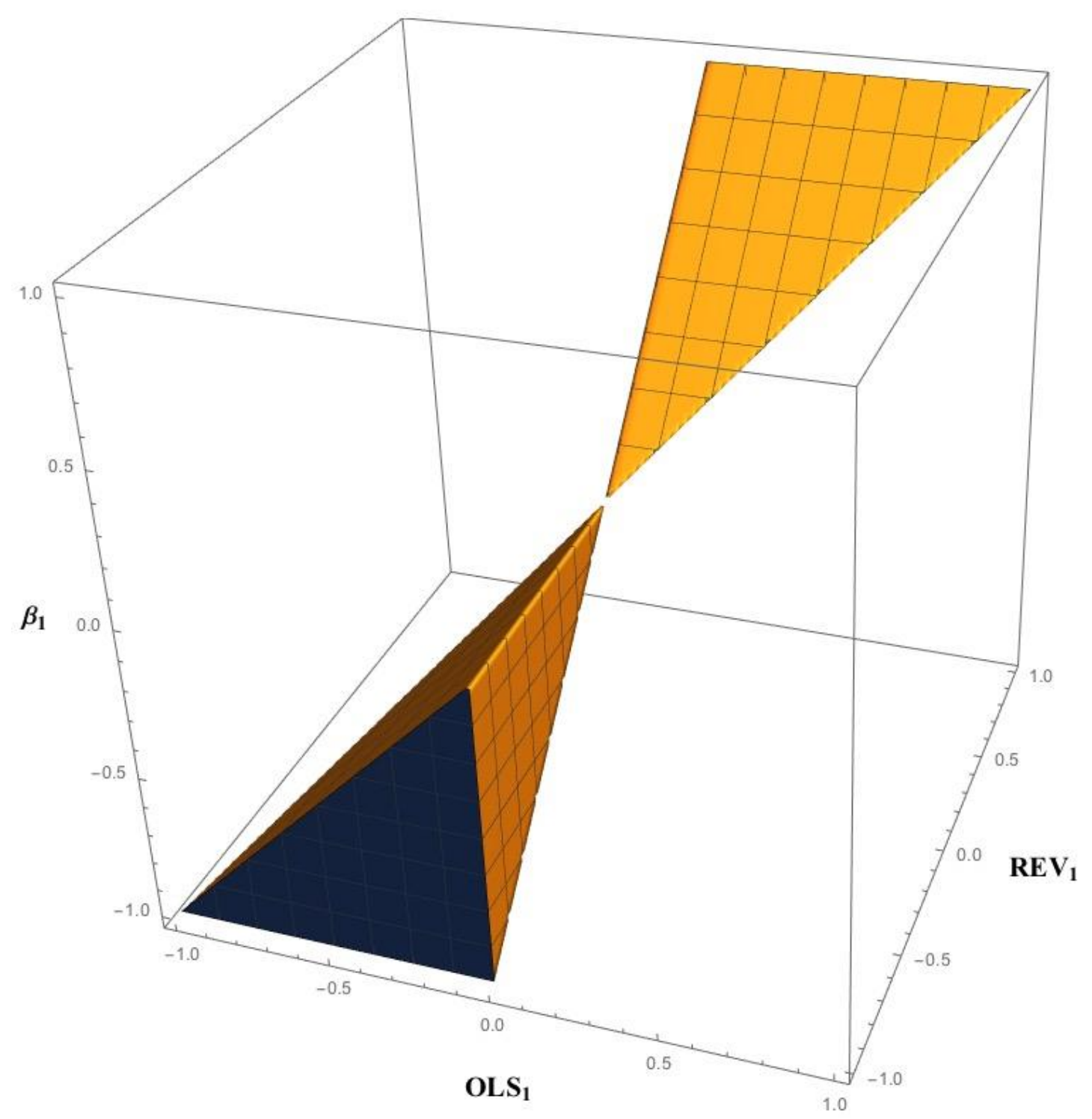




\section{Figure 3. Mathematica screen shot The Nonparametric Roy Model}

$\ln [1]:=$ Get ["http://economicreasoning.com"]

\section{Define employment rate $p$ and average human capital $S$}

$\ln [2]:=\mathbf{p}\left[\sigma_{-}\right.$, meangap_ $]=\int_{-\infty}^{\infty} \int_{-\infty}^{\sigma h-m e a n g a p} f[h, r] d l d \mathrm{~d} h$;

$\mathrm{S}\left[\sigma_{-}\right.$, meangap_ $]=\frac{\int_{-\infty}^{\infty} \int_{-\infty}^{\sigma h-\text { meangap }} \mathrm{h} f[\mathrm{~h}, \mathrm{r}] \mathrm{dl} \mathrm{dlh}}{\mathrm{p}[\sigma, \text { meangap }]} ;$

SkillImpact $=\frac{\mathbb{d S}[\sigma, \mu \mathrm{R}-\mu \mathrm{w}]}{\mathrm{d} \mathrm{x}}$;

Experiment: increase $\sigma$ but keep employment constant

$\ln [4]:=$ DefineExperiment $=\left\{\frac{d \mathrm{p}[\sigma, \mu \mathrm{R}-\mu \mathrm{w}]}{\mathrm{dx}}=\frac{\mathrm{d} \mu \mathrm{w}}{\mathrm{dx}}=0, \frac{\mathbb{d} \sigma}{\mathrm{d} x}>0\right\} ;$

\section{Result for Employment Constant}

$\ln [5]:=$ TheoryGuru $[\{$ DefineExperiment, $\mathrm{p}[\sigma, \mu \mathrm{R}-\mu \mathrm{w}] \geq 0$,

$$
\begin{aligned}
& \frac{\int_{-\infty}^{\infty} h^{2} f[h, \mu w-\mu R+h \sigma] d h}{\int_{-\infty}^{\infty} f[h, \mu w-\mu R+h \sigma] d h}>\left(\frac{\int_{-\infty}^{\infty} h f[h, \mu w-\mu R+h \sigma] d h}{\int_{-\infty}^{\infty} f[h, \mu w-\mu R+h \sigma] d h}\right)^{2}, \\
& \left.\int_{-\infty}^{\infty} f[h, \mu w-\mu R+h \sigma] d h>0\right\},
\end{aligned}
$$

Skillimpact $>0]$
TheoryGuru 6.3 Dashboard Most recent function: TheoryGuru Most recent method: Contradiction Most recent conclusion:

System dimensions:

System status:

Show space

Show unnecessary assumptions

Show instance

Solve equations only

Show assumptions in column

Deduce univariate hypotheses 


\section{Bibliography}

Ackermann, Wilhelm. Solvable Cases of the Decision Problem. Amsterdam: NorthHolland Publishing Company, 1954.

Anai, Hirokazu, and Hitoshi Yanami. "SyNRAC: A Maple-Package for Solving Real Algebraic Constraints." In Computational Science - ICCS 2003, edited by Peter M.A. Sloot, David Abramson, Alexander V. Bogdanov, Jack J. Dongarra, Albert Y. Zomaya and Yuriy E. Gorbachev, 828-837. 2003.

Arai, Noriko H., Hidenao Iwane, Takua Matsuzaki, and Hirokazu Anai. "Mathematics by Machine." ISSAC '14 Proceedings of the 39th International Symposium on Symbolic and Algebraic Computation. New York: ACM, 2014. 1-8.

Basu, Saugata, Richard Pollack, and Marie-Francoise Roy. Algorithms in Real Algebraic Geometry. Berlin: Springer-Verlag (as updated at perso.univ-rennes1.fr), 2011.

Borjas, George J. "The Economics of Immigration." Journal of Economic Literature 32, no. 4 (December 1994): 1667-1717.

Bradford, Russell, James H. Davenport, Matthew England, Scott McCallum, and David Wilson. "Truth Table Invariant Cylindrical Algebraic Decomposition." Journal of Symbolic Computation 76 (September 2016): 1-35.

Brown, Christopher W. "Companion to the Tutorial Cylindrical Algebraic Decomposition Presented at ISSAC 2004." June 30, 2004. http://www.usna.edu/Users/cs/wcbrown/research/ISSAC04/handout.pdf.

Brown, Christopher W. "Improved Projection for Cylindrical Algebraic Decomposition." Journal of Symbolic Computation 32, no. 5 (2001): 447-465.

Brown, Christopher W. "On Quantifer Elimination by Virtual Term Substitution." U.S. Naval Academy Computer Science Dept. Technical Report, August 2005.

Brown, Christopher W. "QEPCAD B - a program for computing with semi-algebraic sets using CADs." ACM SIGSAM Bulletin 37, no. 4 (December 2003): 97-108.

Brown, Christopher W., and James H. Davenport. "The Complexity of Quantifier Elimination and Cylindrical Algebraic Decomposition." Proceedings of the 2007 international symposium on symbolic and algebraic computation, 2007: 54-60.

Brown, Donald J., and Felix Kubler. "Refutable Theories of Value." In Computational Aspects of General Equilibrium Theory, by Donald J. Brown and Felix Kubler, 110. Berlin: Springer-Verlag, 2008.

Brown, Donald J., and Rosa L. Matzkin. "Testable Restrictions on the Equilibrium Manifold." Econometrica 64, no. 6 (November 1996): 1249-62.

Bryant, Randal E., Steven German, and Miroslav N. Velev. "Exploiting Positive Equality in a Logic of Equality with Uninterpreted Functions." In International Conference on Computer Aided Verification CAV 1999, edited by Nicolas Halbwachs and Doron Peled, 470-482. Berlin: Springer-Verlag, 1999.

Carvajal, Andres, Rahul Deb, James Fenske, and John K.-H. Quah. "A Nonparametric Analysis of Multi-product Oligopolies." Economic Theory 57, no. 2 (October 2014): 253-277.

Caviness, B.F., and J.R. Johnson. "Introduction." In Quantifier Elimination and Cylindrical Algebraic Decomposition, by B.F. Caviness and J.R. Johnson, 1-7. Berlin: Springer-Verlag, 1998.

Chambers, Christopher P., and Federico Echenique. Revealed Preference Theory. Cambridge: Cambridge University Press, 2016. 
Chen, Changbo, and Marc Moreno Maza. "Quantifier elimination by cylindrical algebraic decomposition based on regular chains." Journal of Symbolic Computation 75 (July-August 2016): 74-93.

Chen, Changbo, and Marc Moreno Maza. "Simplification of Cylindrical Algebraic Formulas." In Computer Algebra in Scientific Computing, by Vladimir P. Gerdt, Wolfram Koepf, Werner M. Seiler and Evgenii V. Vorozhtsov, 119-34. Cham, Switzerland: Springer International Publishing, 2015.

Collins, George E. "Efficient Quantifier Elimination for Elementary Algebra." Seminar presentation at Stanford University. 1973.

—. "Quantifier Elimination for Real Closed Fields by Cylindric Algebraic Decomposition." Second GI Conference on Automata Theory and Formal Languages. Berlin: Springer-Verlag, 1975. 134-83.

Corzilius, Florian, Ulrich Loup, Sebastian Junges, and Erika Ábrahám. "SMT-RAT: An SMT-Compliant Nonlinear Real Arithmetic Toolbox." In Theory and Applications of Satisfiability Testing - SAT 2012, edited by Alessandro Cimatti and Sebastiani Roberto, 442-448. 2012.

Cox, David A., John B. Little, and Donal O'Shea. Ideals, Varieties, and Algorithms. New York: Springer, 2007.

Davenport, J.H., Y. Siret, and E. Tournier. Computer Algebra: Systems and Algorithms for Algebraic Computation. London: Academic Press, 1988.

Davenport, James H., and Matthew England. "Recent Advances in Real Geometric Reasoning." In Automated Deduction in Geometry, by Francisco Botana and Pedro Quaresma, 37-52. Springer-Verlag, 2015.

De Morgan, Augustus. "On the syllogism: V, and on various points of the onymatic system." Transactions of the Cambridge Philosophical Society 10 (1862): 428-87.

Dennis, Louise A., Michael Fisher, Matthew P. Webster, and Rafael H. Bordini. "Model checking agent programming languages." Automated Software Engineering 19, no. 1 (2012): 5-63.

Dolzmann, Andreas, and Thomas Sturm. "REDLOG: Computer Algebra Meets Computer Logic." SIGSAM Bull 31, no. 2 (June 1997): 2-9.

England, Matthew, and James H. Davenport. "The Complexity of Cylindrical Algebraic Decomposition with Respect to Polynomial Degree." In Computer Algebra in Scientific Computing. CASC 2016, edited by Gerdt Vladimir, Koepf Wolfram, Werner M. Seiler and Evgenii V. Vorozhtsov, 172-192. 2016.

Fisher, Franklin M. The identification problem in econometrics. New York: McGrawHill, 1966.

Fontaine, Pascal, Mizuhito Ogawa, Thomas Sturm, and Xuan Tung Vu. "Subtropical Satisfiability." In Frontiers of Combining Systems. FroCoS 2017, edited by Clare Dixon and Marcelo Finger, 189-206. 2017.

Friedman, Milton. A Theory of the Consumption Function. Princeton, NJ: Princeton University Press (for NBER), 1957.

Frisch, Ragnar. Statistical Confluence Analysis by Means of Complete Regression Systems. Oslo: University Economic Institute, 1934.

Grigor'ev, D. Yu. "Complexity of deciding Tarski algebra." Journal of Symbolic Computation 5, no. 1 (February-April 1988): 65-108.

Gronau, Reuben. "Wage Comparisons-A Selectivity Bias." Journal of Political Economy 82, no. 6 (March 1974): 1119-1143. 
Heckman, James J. "Sample Selection Bias as a Specification Error." Econometrica 47, no. 1 (January 1979): 153-161.

Heckman, James J., and Bo E. Honore. "The Empirical Content of the Roy Model." Econometrica 58, no. 5 (September 1990): 1121-1149.

Heckman, James J., and Guilherme Sedlacek. "Heterogeneity, Aggregation, and Market Wage Functions: An Empirical Model of Self-Selection in the Labor Market." Journal of Political Economy 93, no. 6 (December 1985): 1077-1125.

Jovanović, Dejan, and B. Dutertre. "LibPoly: A library for reasoning about polynomials." In Proceedings of the 15th International Workshop on Satisfiability Modulo Theories (SMT 2017). 2017.

Jovanović, Dejan, and Leonardo de Moura. "Solving Non-linear Arithmetic." In Automated Reasoning. IJCAR 2012, edited by Bernhard Gramlich, Dale Miller and Uli Sattler, 339-354. 2012.

Kauers, Manuel. "How to Use Cylindrical Algebraic Decomposition." Seminaire Lotharingien de Combinatoire 65 (2011): 1-16.

Keane, Michael, Robert Moffitt, and David Runkle. "Real Wages over the Business Cycle Estimating the Impact of Heterogeneity with Micro Data." Journal of Political Economy 96, no. 6 (December 1988): 1232-66.

Kerber, Manfred, Christoph Lange, and Colin Rowat. "An Introduction to Mechanized Reasoning." Journal of Mathematical Economics 66 (October 2016): 26-39.

Klepper, Steven, and Edward E. Leamer. "Consistent Sets of Estimates for Regressions with Errors in All Variables." Econometrica 52, no. 1 (January 1984): 163-183.

Kroening, Daniel, and Ofer Strichman. Decision Procedures: An Algorithmic Point of View. Berlin: Springer-Verlag, 2008.

Levi, Maurice D. "Errors in the Variables Bias in the Presence of Correctly Measured Variables." Econometrica 41, no. 5 (September 1973): 985-986.

Li, Xiaoliang, and Dongming Wang. "Computing Equilibria of Semi-algebraic Economies Using Triangular Decomposition and Real Solution Classification." Journal of Mathematical Economics 54 (October 2014): 48-58.

Mourifie, Ismael, Marc Henry, and Romuald Meango. "Sharp Bounds for the Roy Model." arXiv.org. September 2017. https://arxiv.org/abs/1709.09284.

Mulligan, Casey B. "Automated Economic Reasoning with Quantifier Elimination." NBER working paper, no. 22922 (December 2016).

Mulligan, Casey B., and Kevin K. Tsui. "The Upside-down Economics of Regulated and Otherwise Rigid Prices." NBER working paper, no. 22305 (June 2016).

Mulligan, Casey B., and Yona Rubinstein. "Selection, Investment, and Women's Relative Wages over Time." Quarterly Journal of Economics 123, no. 3 (August 2008): 1061-110.

Mulligan, Casey B., James H. Davenport, and Matthew England. "TheoryGuru: A Mathematica Package to apply Quantifier Elimination Technology to Economics." manuscript, University of Chicago, May 2018.

Mulligan, Casey B., Russell Bradford, James H. Davenport, Matthew England, and Zak Tonks. "Non-linear Real Arithmetic Benchmarks derived from Automated Reasoning in Economics." NBER working paper, May 2018a.

—. "Quantifier Elimination for Reasoning in Economics." arXiv.org. April 2018b. Passmore, Grant Olney, and Denis Ignatovich. "Formal Verification of Financial Algorithms." In Automated Deduction - CADE 26, edited by Leonardo de Moura, 26-41. Cham: Springer, 2017. 
Renegar, James. "Recent Progress on the Complexity of the Decision Problem for the Reals." In Quantifier Elimination and Cylindrical Algebraic Decomposition, by B.F. Caviness and J.R. Johnson, 220-41. Berlin: Springer-Verlag, 1998.

Shankar, Natarajan. "Little Engines of Proof." In FME 2002:Formal Methods-Getting IT Right, by Lars-Henrik Eriksson and Peter Alexander Lindsay, 1-20. SpringerVerlag, 2002.

Snyder, Susan K. "Nonparametric Testable Restrictions of Household Behavior." Southern Economic Journal 67, no. 1 (July 2000): 171-185.

Steinhorn, Charles. "Tame Topology and O-Minimal Structures." In Computational Aspects of General Equilibrium Theory, by Donald J. Brown and Felix Kubler, 165-91. Berlin: Springer-Verlag, 2008.

Strzebonski, Adam. "Computation with semialgebraic sets represented by cylindrical algebraic formulas." ISSAC '10 Proceedings of the 35th International Symposium on Symbolic and Algebraic Computation. New York: ACM, 2010. 61-68.

Strzeboński, Adam. "Cylindrical algebraic decomposition using local projections." Journal of Symbolic Computation 76 (September-October 2016): 36-64.

Sturm, Thomas. "A Survey of Some Methods for Real Quantifier Elimination, Decision, and Satisfiability and Their Applications." Mathematics in Computer Science 11, no. 3 (December 2017): 483-502.

Tadjouddine, Emmanuel M., Frank Guerin, and Wamberto Vasconcelos. "Abstracting and Verifying Strategy-Proofness for Auction Mechanisms." In Declarative Agent Languages and Technologies VI. DALT 2008., edited by Matteo Baldoni, Tran Cao Son, M. Birna van Riemsdijk and Michael Winikoff, 197-214. Berlin: Springer, 2009.

Tamer, Elie. "Partial Identification in Econometrics." Annual Review of Economics 2, no. 1 (January 2010): 167-195.

Tarski, Alfred. A Decision Method for Elementary Algebra and Geometry. Los Angeles: RAND Corporation, 1951.

Weispfenning, Volker. "Quantifier Elimination for Real Algebra — the Quadratic Case and Beyond." Applicable Algebra in Engineering, Communication and Computing 8, no. 2 (1997): 85-101.

Weispfenning, Volker. "The complexity of linear problems in fields." Journal of Symbolic Computation 5, no. 1 (1988): 3-27. 